\title{
Amphetamine differentially affects temporally spaced bar pressing and collateral water drinking'
}

\author{
Evalyn F. Segal and Sam A. Deadwyler \\ SAN DIEGO STATE COLLEGE
}

\begin{abstract}
Abstraet
Rats trained to space bar presses $20 \mathrm{sec}$. apart for dry food reinforcement engaged in water drinking during the intervals between bar presses. When $1.5 \mathrm{mg} / \mathrm{kg}$ dl-amphetamine was administered, bar pressing rate increased while the incidence of water drinking decreased. Drinking occurred only after the delivery of food pellets.
\end{abstract}

\section{Problem}

Segal \& Holloway (1963) found that rats whose key presses were reinforced on a DRL $20 \mathrm{sec}$. dry food reinforcement schedule spent most of the time between key presses drinking from a water trough in the conditioning chamber. They concluded that water drinking mediated timing behavior. But it is not clear whether the drinking is mediational in the strong sense of supplying the discriminative stimulus for the timing response, or only in the weak sense of intervening between, and competing with, key pressing, thus preventing its too frequent occurrence. To avoid confusion, we will hereafter call collateral any behavior intervening between instances of the timing response, and will reserve the term mediating for cases where it is clear that the collateral behavior generates the discriminative stimulus for the timing response, that is; provides some unique cue correlated with the lapse of the requisite time interval.

Another form of collateral behavior can be generated by using a concurrent reinforcement schedule for a second response, along with the spaced response schedule. Segal (1961) studied the performance of rats trained on a concurrent DRL $16 \mathrm{sec}$. VI $1 \mathrm{~min}$. schedule, one schedule being associated with one lever and the other schedule with a second lever. The subjects of Segal's (1961) study behaved much the same as the subjects of Segal \& Holloway's (1963) study, in that they alternated regularly between single, isolated DRL responses, and long bursts of collateral responding (bar pressing on the VI lever, in one case, and licking from the water trough, in the other). When the rats on concurrent DRL VI were given 1.0 or $1.5 \mathrm{mg} / \mathrm{kg}$ of dl-amphetamine they showed roughly proportional increases in the rates of the DRL and collateral VI responses (Segal, 1962). The present experiment was performed (1) to determine if amphetamine would have similarly proportional effects on spaced responding associated with collateral water drinking, and (2) to attempt to clarify the nature of the relation between timing behavior and collateral water drinking.
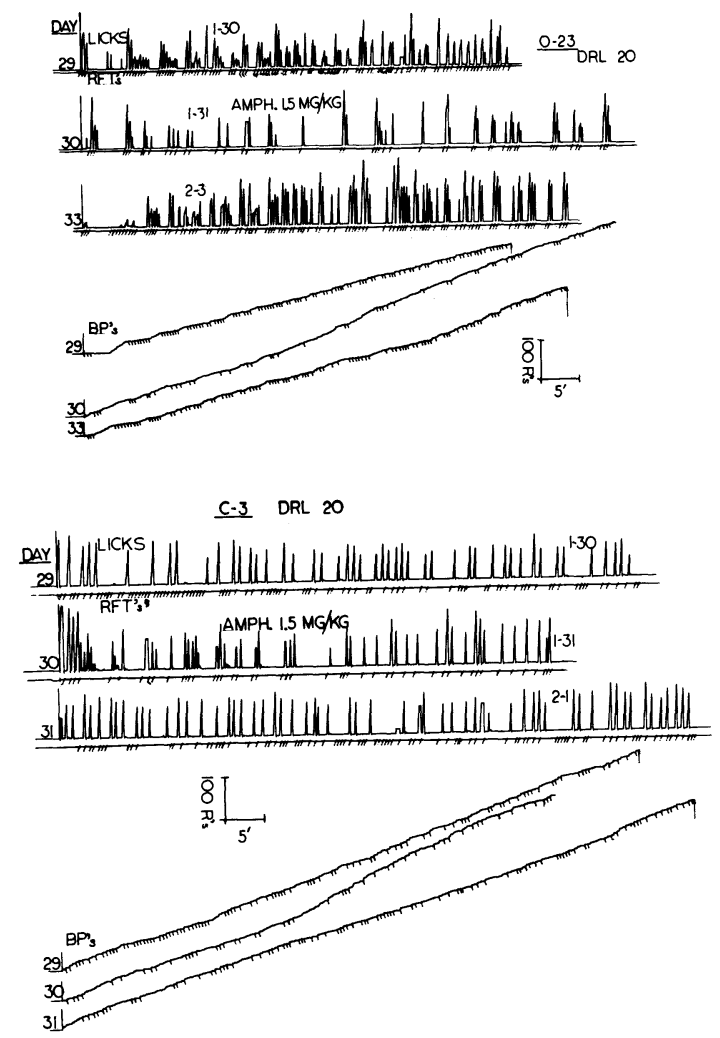

Fig. 1. Water drinking and bar pressing on DRL 20. Upper part: S 0-23; lower part: SC-3. Records labelled "Licks" show cumulative water licks between bar presses; every bar press reset the record. Event tracings below licking records show reinforcements. Record labelled "BP's" show cumulative bar presses from the same sessions, and, as diagonal deflections, reinforcements. Owing to a recording failure, the third session following drug is shown for $0-23$, instead of the first.

\section{Method}

Ss were two adult, male, albino rats, maintained at $80 \%$ of ad lib. weight. Water was always available. They were given 100 reinforcements daily on non-drug days, and 50 reinforcements on the single drug day, on a DRL $20 \mathrm{sec}$. schedule for bar pressing. The reinforcers were $45 \mathrm{mg}$ Noyes pellets. Immediately before each rat's 30th session it was injected intraperitoneally with 1.5 $\mathrm{mg} / \mathrm{kg}$ of dl-amphetamine sulfate dissolved in $0.9 \%$ saline. 


\section{Results}

Figure 1 shows the results of the drug session, compared with the immediately preceding and succeeding sessions. Bar pressing rates increased under drug, and then declined to pre-drug levels on the day following drug. Accompanying the increase in bar pressing rates on the drug day, there was a decline in the frequency of food reinforcement. (Most bar presses were spaced too close together to earn reinforcement.) Finally, a close correlation between the arrival of food and the occurrence of water drinking became evident. The rarity of reinforcements on the drug day makes this correlation easy to see, but the correlation is also evident, in attenuated form, on non-drug days. Under amphetamine, virtually every reinforcement was followed by drinking, and no drinking occurred that was not initiated by reinforcement. On non-drug days, some reinforcements were not followed by drinking, but few drinking episodes were not initiated by reinforcement. (All pellets were consumed on the drug day.)

\section{Diseussion}

On a DRL schedule, the effect of amphetamine on collateral water drinking was unlike its effect on collateral VI bar pressing. Amphetamine lowered the overall incidence of drinking, but raised the rate of VI responding. Amphetamine also increased the probability that a food pellet would initiate drinking.

The results suggest that the facilitative effect of water drinking on timing behavior (Segal \& Holloway, 1963) is mainly a fortuitous by-product of rats' tendency to drink following ingestion of dry food on intermittent schedules of food delivery. Such drinking does not appear to reflect a mediational process in the more restrictive sense. On the other hand, the collateral VIbar pressing under concurrent DRL VI might be mediational, inasmuch as both DRL and VI response rates increased proportionally under amphetamine (Segal, 1962). If the emission of a given amount of VI bar pressing provided the discriminative stimulus for switching to the DRL lever, then the VI-DRL response chain remained intact under drug, and was simply run off faster than on nondrug days.

\section{References}

SEGAL, EVALYN F. Behavioral interaction under concurrent spaced-responding, variable-interval schedules of reinforcement. J. exp. Anal.Beh., 1961, 4, 263-266.

SEGAL, EVALYN F. Effects of dl-amphetamine under concurrent VI DRL reinforcement. J. exp. Anal. Beh., 1962, 5, 105-112.

SEGAL,EVALYN F., \& HOLLOWAY, S. M. Timing behavior in rats with water drinking as a mediator. Science, 1963, 140, 888-889.

\section{Note}

1. Supported by grants NSF G 18132, NSF GB 1605, NIMH 8505, and an NSF undergraduate research training grant. 